\title{
Cancer gene therapy 2020: highlights from a challenging year
}

\author{
Georgios Giamas $\mathbb{D}^{1} \cdot$ Teresa Gagliano $\mathbb{1}^{2}$
}

Received: 11 March 2021 / Revised: 4 April 2021 / Accepted: 13 April 2021 / Published online: 7 May 2021

(C) The Author(s), under exclusive licence to Springer Nature America, Inc. 2021

During 2020, like everything else, cancer research has been challenged by the global Covid-19 pandemic [1, 2]. Although most of the laboratories worldwide had a period of forced stop, still scientists have found ways to cope and adapt to the rapidly changing circumstances and remain focused and productive during these challenging times.

Being perfectly aware of the difficulties that investigators have been facing, Cancer Gene Therapy (CGT) has adjusted the peer review process accordingly (i) by extending the revision timelines, (ii) by being realistic in the requests for technically challenging and time-consuming additional experiments and (iii) by demanding to tone down conclusions where definite supporting data were not available. Despite these conditions, the journal's priorities to safeguard the quality and sustain the integrity of the published scientific work were not affected.

The researchers' resilience is embodied in the fascinating research that has been published in CGT over the last year and that has captured the interest of the scientific community. Here, we highlight some of the articles that cover different aspects of current cutting-edge research including the development of organoids as cancer models, novel methods like new drug delivery systems as well as innovative applications of the zebrafish model.

Georgios Giamas

G.Giamas@sussex.ac.uk

$\bowtie$ Teresa Gagliano

teresa.gagliano@uniud.it

1 Department of Biochemistry and Biomedicine, School of Life Sciences, University of Sussex, Falmer, Brighton, UK

2 Department of Medical Science, University of Udine, Udine, Italy

\section{Identification of new therapeutic targets for AML}

One of the goals in cancer research is discovering oncogenic drivers, which often include cancer's mutational status. However, it is now well established that the mechanisms that lead to genetic mutations should be considered pivotal factors in neoplastic transformation. A perfect example of this approach is the work published by Ruckert et al. Acute myeloid leukemia (AML) is a hematopoietic malignancy with poor prognosis, particularly in the elderly population [3]. HUWE1, an E3 ubiquitin ligase involved in several cellular processes, was identified to be the second most expressed gene in AML, according to a TCGA-based analysis. Using leukemia cells constitutively expressing mutated RAS (KRAS ${ }^{\mathrm{G} 12 \mathrm{~V}}$ ), the authors identified a mechanism by which HUWE1 cooperates with mutated RAS activation and controls the proliferation of cancer cells. In addition, in silico analysis highlighted that expression of HUWE1 correlates with myeloid differentiation-related genes. Their findings suggest that HUWE1 is not only involved in controlling RAS mutated AML cell proliferation but could also be implicated in myeloid differentiation [4].

\section{Signature of AML stem cells}

Another challenge in AML research is to identify new genetic signatures able to detect AML-specific stem cells that are responsible for tumor relapse and therapy resistance [5]. An alternative bioinformatic approach has been employed by $\mathrm{Li}$ et al. In their manuscript, using Monte Carlo feature selection combined with machine learning algorithms, the authors developed a method to identify gene expression features that are specific for stem cells. The identified genes $(n=17)$ can be considered as specific biomarkers of leukemia stem cells and may have a fundamental impact on future research and therapy design [6]. 


\section{Ferroptosis, a new pathway of cell death}

Cell signaling that regulates cell death has been the central core of cancer drug discovery for decades. Along with necrosis and apoptosis, it is currently known that cells can use several alternative routes to trigger cell death [7]. In recent years, another type of programmed cell death, namely ferroptosis, has been described, which is accompanied by a large amount of iron accumulation and lipid peroxidation [8].

The review by Dai et al. [9], describes in a thorough and comprehensive way the transcription factors involved in ferroptosis that can be targeted in different cancer types. The same group has also studied autophagy-dependent ferroptosis. Inhibition of the mammalian target of rapamycin kinase (mTOR) with rapamycin is used to trigger autophagy [10]. The authors identified a role glutathione peroxidase 4 , an antioxidant enzyme, in modulating autophagy-dependent ferroptosis in pancreatic cancer cells. The results demonstrated that treatment with rapamycin induces GPX4 degradation and consequently ferroptosis. In addition, researchers found that genetic silencing of GPX4 was sufficient to trigger autophagy-dependent ferroptosis [11]. These findings shed light on the mechanisms of stress response during cell death and suggest possible targets for cancer therapy.

\section{A central role for PI3K in cancer research}

The critical role of the PI3K/AKT/mTOR pathway in cancer has been demonstrated by several studies [12]. However, the role of PIK3 as a prognostic tool in advanced malignancies has not been fully demonstrated. In the review by Willis et al. [13], the authors analyzed the role of PIK3CA mutations in cancer and patient management and conclude that future PIK3CA-targeted therapy will rely on a better understanding of the PI3K/AKT/mTOR signaling pathway, and on the development of target-specific inhibitors.

\section{Oncolytic viruses, new frontiers for cancer treatment}

Oncolytic viruses (OVs) are the object of intense research in both basic science and clinical trials. OVs should infect malignant cells, albeit not all the mechanisms and strategies that can drive this selectivity have been elucidated. HulinCurtis and co-authors identified several peptides that bind to Folate receptor $\alpha(\mathrm{FR} \alpha)$ as a potential way to target the delivery of human adenoviral oncolytic virotherapies. FR $\alpha$ is a membrane protein involved in folate metabolism, with relatively low expression in normal cells, which is however upregulated in cancer cells [14]. The identified FR $\alpha$-binding peptides were genetically introduced in human adenoviruses and showed specificity for cancer cells in vivo. This study represents a promising starting point for improving targeted OVs, however, the study did not improve the delivery of OVs carrying the peptides sequences to cancer cells, compared to wild-type OVs [15].

\section{Adapting Zebrafish to create a flexible model for cancer research}

On the same topic, Mealiea et al., set up an interesting experimental system to monitor the effects of OVs by adapting a zebrafish tumor xenograft model. By exploiting the transparency of zebrafish during the embryonic stage and by combining different zebrafish strains harboring fluorescent immune and endothelial cells, the authors were able to investigate in real-time important events, such as angiogenesis and tumor formation, related to tumor formation and also associated with how tumors respond to OVs treatment [16]. This system may represent a useful model for future OVs research and therapy design.

\section{Organoids, tumor in a dish}

Using adequate models to identify new therapeutic molecules that can control tumor growth is of fundamental importance. Tumor organoids can be used to recapitulate in vitro the pathological features of a disease, including its response to treatment. With this in mind, Chen et al., set up an organoid-based drug screening assay to identify inhibitors for the treatment of endometrial cancer. The authors screened a small library of molecules targeting epigenetic factors. They identified menin-MLL inhibitors that were able to reduce the growth of mouse organoids. MEN1 gene was found to be upregulated in endometrial cancer and MEN1 levels correlated with poor prognosis [17], suggesting it as a potential new target for endometrial cancer treatment.

\section{Manipulating immune cells to improve cancer therapy}

Another useful tool for targeting cancer cells is the chimeric antigen receptors (CARs) that make $\mathrm{T}$ lymphocytes tumorspecific. CAR-T cells have shown promising results and have been recently approved by FDA and EMA for lymphoma and leukemia treatment [18]. However, there are still several issues that need to be addressed before this approach could see a wider application. In the work by Papathanasiou et al., the authors described the challenges and opportunities behind this therapeutic approach [19]. 


\section{Methods to overcome drug resistance}

Despite the overall progress, there are still cancer types for which medical therapy remains extremely challenging, due to the lack of appropriate targeting molecules and/or intrinsic and acquired resistance processes. A perfect example of such malignancies is neuroendocrine tumors (NETs), a relatively rare disease arising from the neuroendocrine cells spread in the normal epithelium [20]. Si et al., aimed to develop an antibody-drug conjugated (ADC) to treat NETs. As a result, they produced an antibody conjugated with antimitotic auristatin $\mathrm{E}$ that was able to target the two extracellular domains of the somatostatin receptor 2 (SSTR2), which has strong specificity for NET cells, promoting their death. Ultimately, this new approach might be used to improve the therapeutic management of NETs in the clinic [21].

\section{Summary}

Overall, despite the unprecedented difficulties that have shaped 2020, it is remarkable and encouraging to witness the continuous and fruitful efforts of cancer researchers and scientists around the world who remain committed to scientific excellence.

\section{Compliance with ethical standards}

Conflict of interest Georgios Giamas is Editor in Chief and Teresa Gagliano is an Associate Editor of CGT.

Publisher's note Springer Nature remains neutral with regard to jurisdictional claims in published maps and institutional affiliations.

\section{References}

1. Weiner DL, Balasubramaniam V, Shah SI, Javier JR, Pediatric Policy Council. COVID-19 impact on research, lessons learned from COVID-19 research, implications for pediatric research. Pediatr Res. 2020;88:148-50. https://doi.org/10.1038/s41390-020-1006-3.

2. Harris AL. COVID-19 and cancer research. $\mathrm{Br} \mathrm{J}$ Cancer. 2020;123:689-90. https://doi.org/10.1038/s41416-020-0960-1.

3. Shimada A. Hematological malignancies and molecular targeting therapy. Eur J Pharmacol. 2019;862:172641. https://doi.org/10. 1016/j.ejphar.2019.172641.

4. Ruckert MT, Brouwers-Vos AZ, Nagano LFP, Schuringa JJ, Silveira VS. HUWE1 cooperates with RAS activation to control leukemia cell proliferation and human hematopoietic stem cells differentiation fate. Cancer Gene Ther. 2020;27:830-3. https://doi. org/10.1038/s41417-020-0198-3.

5. Guarnerio J, Mendez LM, Asada N, Menon AV, Fung J, Berry K, et al. A non-cell-autonomous role for Pml in the maintenance of leukemia from the niche. Nat Commun. 2018;9:66. https://doi.org/ 10.1038/s41467-017-02427-x.

6. Li J, Lu L, Zhang YH, Xu Y, Liu M, Feng K, et al. Identification of leukemia stem cell expression signatures through Monte Carlo feature selection strategy and support vector machine. Cancer Gene Ther. 2020;27:56-69. https://doi.org/10.1038/s41417-0190105-y.

7. Chen X, Kang R, Kroemer G, Tang D. Broadening horizons: the role of ferroptosis in cancer. Nat Rev Clin Oncol. 2021. https:// doi.org/10.1038/s41571-020-00462-0.

8. Li J, Cao F, Yin HL, Huang ZJ, Lin ZT, Mao N, et al. Ferroptosis: past, present and future. Cell Death Dis. 2020;11:88. https://doi. org/10.1038/s41419-020-2298-2.

9. Dai C, Chen X, Li J, Comish P, Kang R, Tang D. Transcription factors in ferroptotic cell death. Cancer Gene Ther. 2020;27:645-56. https://doi.org/10.1038/s41417-020-0170-2.

10. Cristofani R, Montagnani Marelli M, Cicardi ME, Fontana F, Marzagalli M, et al. Dual role of autophagy on docetaxelsensitivity in prostate cancer cells. Cell Death Dis. 2018;9:889. https://doi.org/10.1038/s41419-018-0866-5.

11. Liu Y, Wang Y, Liu J, Kang R, Tang D. Interplay between MTOR and GPX4 signaling modulates autophagy-dependent ferroptotic cancer cell death. Cancer Gene Ther. 2021;28:55-63. https://doi. org/10.1038/s41417-020-0182-y.

12. LoRusso PM. Inhibition of the PI $3 \mathrm{~K} / \mathrm{AKT} / \mathrm{mTOR}$ pathway in solid tumors. J Clin Oncol. 2016;34:3803-15. https://doi.org/10. 1200/JCO.2014.59.0018.

13. Willis O, Choucair K, Alloghbi A, Stanbery L, Mowat R, Charles Brunicardi F, et al. PIK3CA gene aberrancy and role in targeted therapy of solid malignancies. Cancer Gene Ther. 2020;27:634-44. https://doi.org/10.1038/s41417-020-0164-0.

14. Kalli KR, Block MS, Kasi PM, Erskine CL, Hobday TJ, Dietz A, et al. Folate receptor alpha peptide vaccine generates immunity in breast and ovarian cancer patients. Clin Cancer Res. 2018;24:3014-25. https://doi.org/10.1158/1078-0432.CCR-172499.

15. Hulin-Curtis SL, Davies JA, Nestić D, Bates EA, Baker AT, Cunliffe TG, et al. Identification of folate receptor $\alpha(\mathrm{FR} \alpha)$ binding oligopeptides and their evaluation for targeted virotherapy applications. Cancer Gene Ther. 2020;27:785-98. https://doi.org/ 10.1038/s41417-019-0156-0.

16. Mealiea D, Boudreau E, De Silva N, Okamoto L, Ho T, Fish JE, et al. Modeling oncolytic virus dynamics in the tumor microenvironment using zebrafish. Cancer Gene Ther. 2020. https://doi. org/10.1038/s41417-020-0194-7.

17. Chen J, Zhao L, Peng H, Dai S, Quan Y, Wang M, et al. An organoid-based drug screening identified a menin-MLL inhibitor for endometrial cancer through regulating the HIF pathway. Cancer Gene Ther. 2021;28:112-25. https://doi.org/10.1038/ s41417-020-0190-y.

18. Gorovits B, Koren E. Immunogenicity of chimeric antigen receptor T-cell therapeutics. BioDrugs 2019;33:275-84. https:// doi.org/10.1007/s40259-019-00354-5.

19. Papathanasiou MM, Stamatis C, Lakelin M, Farid S, TitchenerHooker N, Shah N, et al. T-cell therapies supply chain: challenges and opportunities? Cancer Gene Ther. 2020;27:799-809. https:// doi.org/10.1038/s41417-019-0157-z.

20. Gagliano T, Brancolini C. Targeting histone deacetylases for combination therapies in neuroendocrine tumors. Cancer Gene Ther. 2020. https://doi.org/10.1038/s41417-020-00260-x.

21. Si Y, Kim S, Ou J, Lu Y, Ernst P, Chen K, et al. Anti-SSTR2 antibody-drug conjugate for neuroendocrine tumor therapy. Cancer Gene Ther. 2020. https://doi.org/10.1038/s41417-020-0196-5. 